\section{Inkomplette Antikörper}

K. Kleesiek ${ }^{1}$, C. Götting ${ }^{2}$, J. Diekmann ${ }^{3}$, J. Dreier ${ }^{4}$ und M. Schmidt ${ }^{5}$

${ }^{1}$ Ehemaliger Direktor des Instituts für Laboratoriums- und Transfusionsmedizin, Herz- und Diabeteszentrum NordrheinWestfalen Ruhr-Universität Bochum, Bad Oeynhausen, Deutschland

${ }^{2}$ MVZ Labor Limbach Nürnberg GmbH, Nürnberg, Deutschland

${ }^{3}$ Institut für Laboratoriums- und Transfusionsmedizin, Herz- und Diabeteszentrum NRW, Bad Oeynhausen, Deutschland

${ }^{4}$ Herz- und Diabeteszentrum Nordrhein-Westfalen; Institut für Laboratoriums- und Transfusionsmedizin, Universitätsklinik der Ruhr-Universität Bochum, Bad Oeynhausen, Deutschland

${ }^{5}$ Institut für Laboratoriums- und Transfusionsmedizin, Universitätsklinik der Ruhr-Universität Bochum, Bad Oeynhausen, Deutschland

Synonym(e) IgG-Antikörper

Englischer Begriff IgG antibodies

Definition Bezeichnung in der Transfusionsmedizin für Antikörper der IgG-Klasse, die ohne Zusatz von AntiHumanglobulin nicht in der Lage sind, im Kochsalzmilieu Erythrozyten, die die entsprechenden Antigene tragen, zu agglutinieren.

Beschreibung Aufgrund ihres Verhaltens bei labortechnischen Nachweismethoden werden in Transfusionsmedizin und Immunhämatologie komplette und inkomplette Antikörper unterschieden. Inkomplette Antikörper sind stets Anti- körper der IgG-Klasse und können beim Antikörpernachweis im Labor im Kochsalzmilieu Erythrozyten, die die korrespondierenden Antigene auf der Zelloberfläche tragen, nicht direkt, sondern nur nach Zusatz eines Sekundärantikörpers (Anti-Humanglobulin) agglutinieren. Komplette Antikörper hingegen gehören immer der IgM-Klasse an und können direkt die Erythrozyten agglutinieren. Diese unterschiedlichen Eigenschaften von Antikörpern, deren Einteilung in komplette und inkomplette Antikörper rein aufgrund ihres labortechnischen Verhaltens erfolgt, beruht auf den Größenunterschieden von IgM- und IgG-Antikörpern und dem $>\mathrm{Ze}$ tapotenzial der Erythrozyten. Das Zetapotenzial ist eine erythrozytenspezifische Eigenschaft, die dazu führt, dass sich Erythrozyten gegenseitig abstoßen und in physiologischem Milieu einen Abstand zueinander von bis zu $300 \AA$ einhalten. Dieser Abstand kann direkt nur von Antikörpern der IgMKlasse, die ein Molekulargewicht von ungefähr $900 \mathrm{kDa}$ aufweisen, überbrückt und somit eine Agglutination der Erythrozyten im Reagenzglas herbeigeführt werden. IgG-Antikörper sind aufgrund ihres geringeren Molekulargewichtes von ungefähr $160 \mathrm{kDa}$ nicht in der Lage, direkt den Abstand von 2 Erythrozyten zu überbrücken und somit ohne Zusatz eines vernetzenden Sekundärantikörpers (Anti-Humanglobulin) eine Agglutination $\mathrm{zu}$ induzieren. Wichtig ist, dass die Unterscheidung von kompletten und inkompletten Antikörpern lediglich aufgrund ihres Verhaltens bei Nachweismethoden im Labor erfolgt und für die Antikörperwirkung in vivo, die nur über die Antigen-Antikörper-Wechselwirkung bestimmt wird, ohne Bedeutung ist.

\section{Literatur}

Eckstein R (2005) Immunhämatologie und Transfusionsmedizin. Urban \& Fischer, München 